\title{
Correction to: Effects of some biological agents on the growth and biochemical parameters of tomato plants infected with Alternaria solani (Ellis \& Martin) Sorauer
}

Gökhan Boyno (D) Semra Demir (D)

Younes Rezaee Danesh (D)

Published online: 15 November 2021

(C) Koninklijke Nederlandse Planteziektenkundige Vereniging 2021

\section{Correction to: Eur J Plant Pathol}

https://doi.org/10.1007/s10658-021-02398-2

Article type needs to be read as "Original Paper" instead of "Review Paper" as it was misclassified during revision at initial submission.

The original article can be found online at https://doi.org/ 10.1007/s10658-021-02398-2.

G. Boyno $\cdot$ S. Demir $(\bowtie)$

Department of Plant Protection, Faculty of Agriculture,

Van Yuzuncu Yil University, Van, Turkey

e-mail: semrademir@yyu.edu.tr

Y. R. Danesh

Department of Plant Protection, Faculty of Agriculture,

Urmia University, Urmia, Iran

Y. R. Danesh

Soil, Fertilizer and Water Resources Central Research Institute, Ankara, Turkey 